\title{
Mapping the Elemental Composition of Ceres and Vesta: Dawn's Gamma Ray and Neutron Detector
}

\author{
T.H. Prettyman, ${ }^{\mathrm{a}}$ W.C. Feldman, ${ }^{\mathrm{a}}$ B.L. Barraclough, ${ }^{\mathrm{a}}$ M.T. Capria, ${ }^{\mathrm{b}}$ A. Coradini, ${ }^{\mathrm{b}}$ D.C. Enemark, ${ }^{\mathrm{a}}$ \\ K.R. Fuller, ${ }^{a}$ D.J. Lawrence, ${ }^{a}$ D.E. Patrick, ${ }^{a}$ C.A. Raymond, ${ }^{c}$ S.A. Storms, ${ }^{a}$ R.L. Williford ${ }^{a}$ \\ ${ }^{a}$ Mail Stop D466, Los Alamos National Laboratory, Los Alamos, New Mexico 87545; \\ ${ }^{\mathrm{b}}$ INAF, Via Fosso del Cavaliere 100, 00133 Rome, Italy; \\ ${ }^{c}$ Mail Stop 183-501, Jet Propulsion Laboratory, 4800 Oak Grove Drive, Pasadena, CA 91109
}

\begin{abstract}
Dawn is a NASA discovery mission that will explore the main belt asteroids (1) Ceres and (4) Vesta. Ceres and Vesta are among the oldest bodies in the solar system and represent very different evolutionary paths. By studying these ancient, complementary asteroids, we will answer fundamental questions about the early solar system and planetary formation processes. The Dawn payload consists of a Framing Camera (FC), a visual and infrared mapping spectrometer (VIR), and a Gamma Ray and Neutron Detector (GRaND). The instruments provide data needed to investigate the structure, geology, mineralogy, and geochemistry of the asteroids. GRaND provides the data for the geochemistry investigation, including maps of most major elements and selected radioactive and trace elements. An updated description of the GRaND instrument is given along with the expected performance of GRaND at Vesta and Ceres. Approaches to combine data from FC, VIR and GRaND are discussed.
\end{abstract}

Keywords: Dawn, asteroids, gamma ray, spectroscopy

\section{INTRODUCTION}

Dawn is a NASA discovery mission that will travel to and orbit main belt asteroids (1) Ceres and (4) Vesta [Russell et al., 2004]. Ceres and Vesta are among the largest asteroids with mean diameters of approximately $1000 \mathrm{~km}$ and 500 $\mathrm{km}$, respectively. Based on spectral reflectance data, the composition of these bodies differs significantly, which suggests that they underwent dissimilar evolutionary processes and may have accreted from different locations in the solar nebula. Vesta is a dry, inner-belt asteroid that differentiated by igneous processes and whose surface consists of pyroxene-bearing basalts similar in composition to the Howardite, Eucrite and Diogenite (HED) meteorites. Ceres is a water-rich, mid-belt object that may have undergone aqueous differentiation at low temperature. The accretion and thermal evolution of bodies in the main asteroid belt is believed to have halted following the formation of Jupiter, whose tidal forcing interrupted further accretion in the belt region. Consequently, it is believed that the main belt asteroids are remnants of the earliest epoch of solar system evolution. By exploring Ceres and Vesta, the Dawn mission will peer into the distant past, providing new information on processes by which the planets formed.

Dawn will launch in 2006 and will travel to the asteroids using solar electric propulsion, arriving at Vesta in 2011 and Ceres in 2016. At each encounter, Dawn will enter into circular, polar mapping orbits to acquire science data. The Dawn payload includes a framing camera (FC), a visible near-infrared mapping spectrometer (VIR), and a gamma ray and neutron detector (GRaND). The framing camera will provide visual imaging data for geomorphology and to map the topography of each asteroid. VIR will provide data on surface mineralogy [Coradini et al., 1998]. GRaND will map the abundance of rock-forming elements, including $\mathrm{O}, \mathrm{Mg}, \mathrm{Al}, \mathrm{Si}, \mathrm{Ca}, \mathrm{Ti}$, and Fe, long-lived radioactive elements, including $\mathrm{K}$, Th, and $\mathrm{U}$, and elements such as $\mathrm{H}, \mathrm{C}$, and $\mathrm{N}$, which are the major constituents of ices. At Ceres, GRaND may be able to distinguish among water, ammonia, methane, and carbon dioxide condensates that may be present on the surface. At Vesta, it may also be possible to determine the abundance of trace elements Gd and Sm.

The science goals that will be addressed by GRaND include:

1) Verify that Vesta is the source of the HED meteorites and determine their geological context. 
2) Characterize the depletion of volatile material in the source material from which the asteroids were formed by measuring the $\mathrm{K} / \mathrm{Th}$ ratio. Is the source material for Vesta and Ceres different?

3) Determine the absolute abundance and spatial distribution of radioactive elements. Did the long-lived radioactive elements contribute significantly to differentiation of the asteroids?

4) Characterize Vesta's basaltic and feldspathic geological units to constrain internal composition and thermal evolution.

5) Determine whether the mantle is exposed in the great south polar crater of Vesta to further constrain Vesta's structure and thermal evolution.

6) Determine whether large-spatial-scale hydrated mineral deposits are present on Vesta, based on hints from meteorite data [Treiman et al., 2004]. How did water influence the evolution of Vesta?

7) Is water cold trapped at the poles of Vesta?

8) Provide constraints on cold aqueous alteration processes on Ceres. Could liquid water still be present on Ceres? Are significant ammonia, methane, or carbon dioxide ice deposits present on the surface? How are they distributed and what are the implications for thermal evolution?

Synthesis of data from all three instruments will provide a coherent picture of the asteroids. Some of the key issues that must be addressed to interpret data acquired by GRaND in light of spectroscopy and imaging data from FC and VIR are discussed here. The Dawn instruments are in the process of being built for flight and will be delivered for integration with the spacecraft in April of 2005. We will also provide an update on the GRaND instrument and its expected performance at Vesta and Ceres. This paper supplements a more detailed description of the instrument by Prettyman et al. [2003].

GRaND measures the spectrum of gamma rays and neutrons produced by galactic cosmic ray interactions and radioactive decay within the top meter of the surface of the asteroids. The quality of data returned by GRaND depends critically on the orbital altitude at which the measurements are made and the duration of the measurements. The baseline mission provides for a low altitude mapping orbit (LAMO) of $200 \mathrm{~km}$ altitude for 50 days at Vesta and a LAMO at Ceres at $700 \mathrm{~km}$ altitude for at least 30 days. We show that this mission scenario is sufficient to acquire data needed to address the aforementioned science questions.

\section{THE GRAND TOUR}

Gamma rays and neutrons can be used to investigate the surface geochemistry of planetary bodies from orbital altitudes. High energy galactic cosmic rays, mainly protons with $\mathrm{GeV}$ energies, interact with nuclei in the surface producing a shower of energetic particles, including neutrons and protons. The neutrons undergo collisions with the nuclei in the surface loosing energy until they escape or achieve thermal equilibrium with the surface material in which they are ultimately absorbed. Characteristic gamma rays for each element in the surface are produced by inelastic collisions with fast neutrons and by neutron capture with thermal neutrons. The leakage spectrum of uncollided gamma rays is shown in Fig. 1a (as lines identified with the target element) for a lunar material [Reedy et al., 1973]. The gamma rays associated with major and minor elements are produced by nuclear reactions. The gamma rays from $\mathrm{K}$, Th, and $\mathrm{U}$ are produced by radioactive decay. A lunar gamma ray spectrum measured by the Lunar Prospector Gamma Ray Spectrometer (LPGRS) BGO sensor is shown for comparison in Fig. 1a [Feldman et al., 2004a; Lawrence et al., 2004].

Neutrons escaping to orbital altitude also contain information about composition. The leakage flux of fast neutrons is sensitive to the average atomic mass of the surface material [Maurice et al., 2000; Gasnault et al., 2001]. Epithermal neutrons with energies between $0.01 \mathrm{eV}$ and $0.5 \mathrm{MeV}$ predominantly undergo elastic collisions. The rate of energy loss for elastic scattering is to a first approximation inversely proportional to atomic mass. Consequently, for surfaces containing hydrogen, carbon, and other light nuclei the epithermal flux is significantly reduced relative to surfaces containing heavier elements [Feldman et al., 2000]. Thermal neutrons undergo neutron capture, and the thermal neutron flux is strongly influenced by the presence of absorbers such as $\mathrm{N}, \mathrm{Cl}, \mathrm{Fe}, \mathrm{Ti}, \mathrm{Gd}$, and $\mathrm{Sm}$. Consequently, thermal neutron measurements provide additional constraints that can be used to determine the abundance of elements that are difficult to measure from the gamma ray spectrum [Elphic et al., 2000]. Measurements of the neutron spectrum 

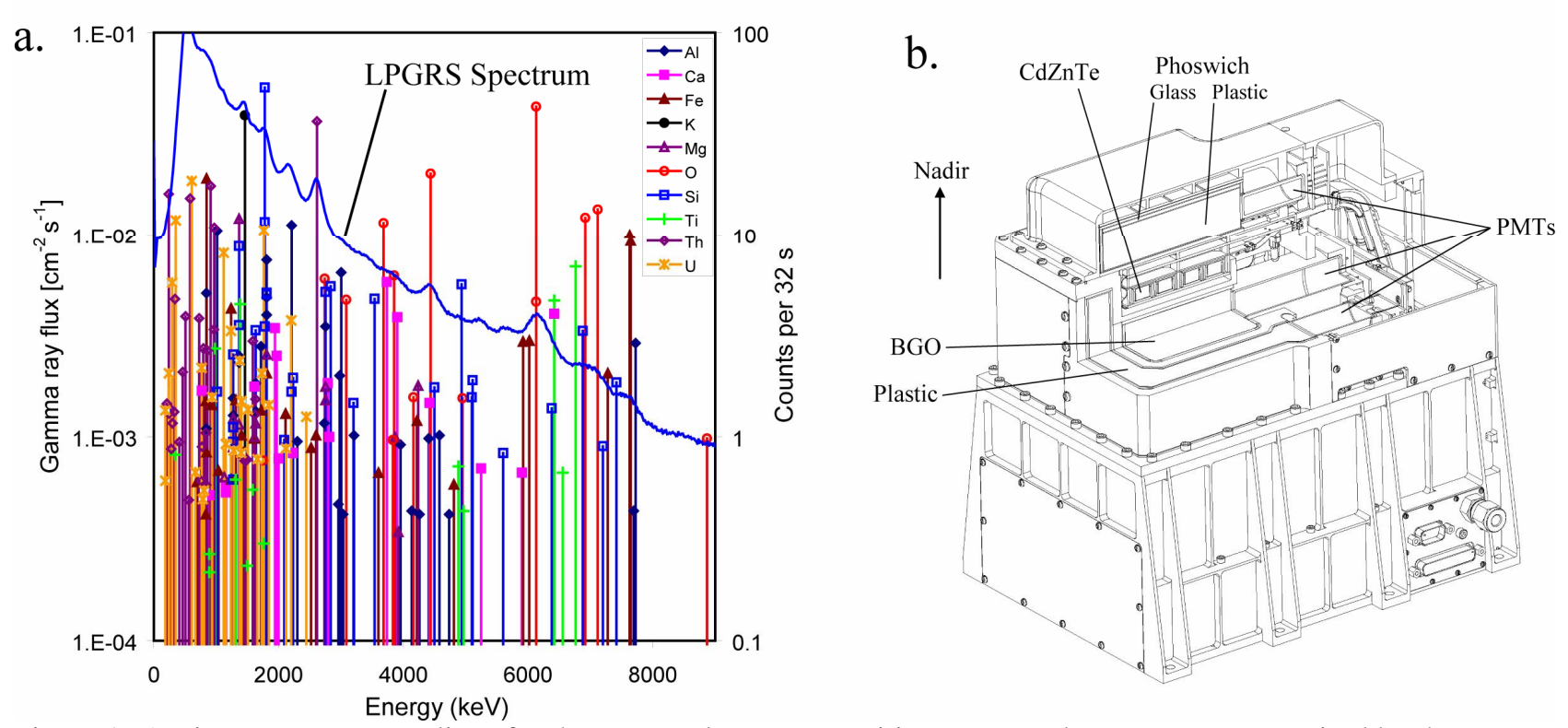

Figure 1. a) Discrete gamma ray lines for the average lunar composition compared to a spectrum acquired by the Lunar Prospector Gamma Ray Spectrometer (LPGRS); and b) a diagram of the Dawn GRaND with a section cut away to show the gamma ray and neutron sensors.
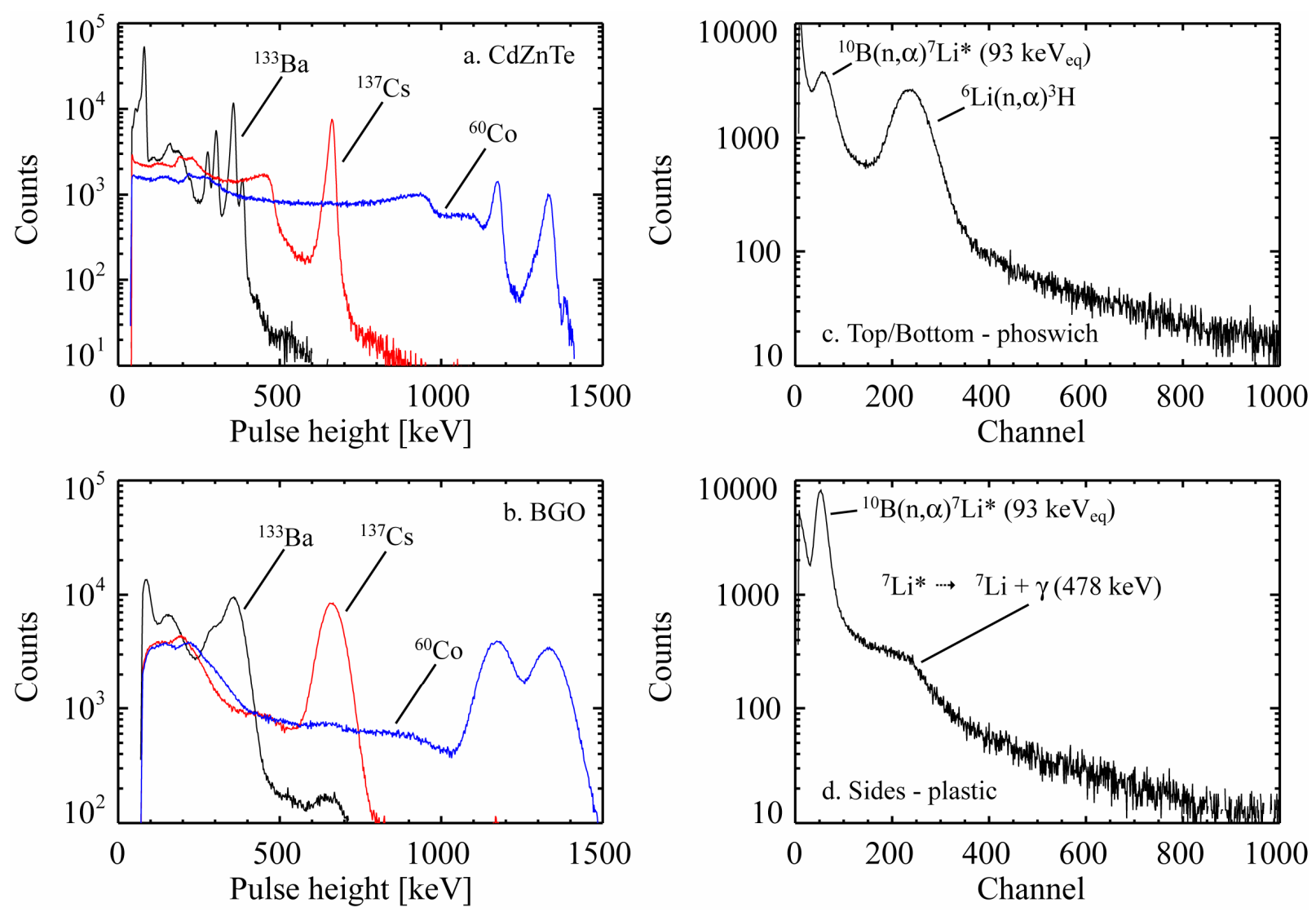

Figure 2. Pulse height spectra acquired for a) a CdZnTe gamma ray sensor; b) a BGO gamma ray sensor; c) a phoswich (top/bottom) neutron sensor; and d) a boron-loaded plastic (left and right side) sensor. 
are also needed in order to interpret gamma ray spectra. The production of gamma rays by neutron capture is proportional to the equilibrium density of neutrons within the surface, which can be determined from measurements of thermal and epithermal leakage flux [Lawrence et al., 2002]. The production of gamma rays by non-elastic reactions is related to the flux of fast neutrons.

The Dawn GRaND consists of 21 sensors designed to measure the spectrum of gamma rays and neutrons originating from Ceres and Vesta. The radiation sensor technology for GRaND was selected based on previous successful missions, including Lunar Prospector and 2001 Mars Odyssey. A new gamma ray sensor technology, CdZnTe, is also being used on GRaND.

A sketch of GRaND is shown in Fig. 1b. A section cut away to reveal a portion of the upper sensor assembly. During acquisition, the orientation of GRaND is constant. The nadir direction is shown for reference. GRaND is mounted directly to the flight deck, instead of deployed on a boom as was LPGRS. Consequently, the sensors are arranged so that the background from the spacecraft can be suppressed or separately measured and subtracted to determine the direct signal from the asteroids.

At the heart of GRaND is a large BGO scintillator $(7.6 \mathrm{~cm} \times 7.6 \mathrm{~cm} \times 5.08 \mathrm{~cm})$, which is roughly the same volume as the LPGRS BGO sensor. The scintillator is optically coupled to a $5.08 \mathrm{~cm}$ diameter phototube. The BGO sensor has high detection efficiency, sufficient for spatial mapping of elemental abundance. Above the BGO sensor (towards nadir) is a $4 \times 4$ array of CdZnTe room-temperature semiconductor sensors [Prettyman et al., 2002]. The BGO acts as an anti-coincidence shield for the CdZnTe array, which will minimize the background caused by gamma rays originating in the spacecraft. The CdZnTe sensors have roughly a factor of four times better pulse height resolution than the BGO and can fully resolve gamma rays from most elements. A comparison between the response of BGO and $\mathrm{CdZnTe}$ flight sensors using three gamma ray calibration sources is shown in Figs. $2 \mathrm{a}$ and $2 \mathrm{~b}$. The ability to resolve gamma ray peaks greatly simplifies the analysis of elemental abundance; however, because the CdZnTe has relatively low detection efficiency, the array will provide whole asteroid and regional abundances. The data acquired by the CdZnTe array will also be used to calibrate the BGO sensor so that elements that do not have well-resolved gamma rays can be mapped with high spatial resolution.

The neutron sensors surround the gamma ray sensors and also act as an anti-coincidence shield to reject energetic particle events. Above and below the gamma ray sensors are two phoswich ("phosphor sandwiches") sensors that are optically coupled to $2.54 \mathrm{~cm}$ diameter phototubes. To the left and right of the gamma ray sensors are two L-shaped plastic scintillators, also coupled to $2.54 \mathrm{~cm}$ diameter tubes. The nadir facing phoswich sensor is well-shielded from the spacecraft and will provide most of the neutron science return. Each of the phoswiches consists of a ${ }^{6} \mathrm{Li}$-loaded, scintillating glass plate that is optically coupled to a boron-loaded plastic scintillator. Both the glass and the plastic scintillate in response to radiation interactions. The phototube views the glass through the plastic. The plastic scintillator and phototube are wrapped in a $0.46 \mathrm{~mm}$ thick $\mathrm{Gd}$ foil, which absorbs thermal neutrons. The Li-loaded glass plate for the top phoswich is exposed to thermal neutrons from the asteroid. Thermal neutrons interact with the plate via a nuclear reaction, ${ }^{6} \mathrm{Li}(\mathrm{n}, \alpha)^{3} \mathrm{H}$. The energetic reaction products, an alpha particle and a triton, excite the glass producing light, which is detected by the phototube. The glass strongly absorbs thermal neutrons so that the plastic scintillator only sees epithermal and fast neutrons from the asteroid. These interact with the plastic, loosing energy until they escape or are absorbed via the ${ }^{10} \mathrm{~B}(\mathrm{n}, \alpha)^{7} \mathrm{Li}^{*}$ reaction. The products of this reaction produce light that is also detected by the phototube.

Signals from the glass and the plastic are separated by the amount of light observed by the phototube. Fig. $2 \mathrm{c}$ shows a pulse height spectrum acquired for one of the flight phoswich sensors for a neutron source with an energy spectrum that is similar to that produced by a planetary surface. Two well-resolved peaks are observed. The lower peak in the spectrum corresponds to epithermal neutrons interacting with the ${ }^{10} \mathrm{~B}$ in the plastic. The higher-amplitude peak corresponds to thermal and epithermal neutrons that interact with the ${ }^{6} \mathrm{Li}$ in the plastic. The peak areas can be measured and subtracted as described in Prettyman et al. [2003] to separately determine the thermal and epithermal neutron count rates. Because the decay times for light production are very different in the plastic and glass, time domain discrimination will also be used to separate events from the plastic and the glass. Fast neutrons are detected in the plastic by the characteristic double pulse coincidence caused by fast neutron elastic collisions with hydrogen followed later by absorption of the neutron by ${ }^{10} \mathrm{~B}$. 
The side L-shaped plastic scintillators are primarily intended for cosmic ray suppression; however, they are boronloaded and help shield the nadir facing phoswich from neutrons originating in the spacecraft. They are also capable of neutron spectroscopy and will return data useful for analysis of surface composition. A pulse height spectrum for one of the flight L-shaped scintillators is shown in Fig. 2d. Despite their unusual geometry, the pulse height resolution for the $93 \mathrm{keV}$ peak associated with the ${ }^{10} \mathrm{~B}(\mathrm{n}, \alpha)$ reaction is slightly better than that of the phoswich sensors.

Overall, GRaND is a relatively simple instrument that delivers significant science. There are three basic modes of operation and no moving parts. The telemetry rate is relatively low, $3.1 \mathrm{kbps}$. The mass of the instrument is approximately $10 \mathrm{~kg}$ and the power consumption is $11 \mathrm{~W}$.

\section{EXPERIENCES FROM LUNAR PROSPECTOR AND MARS ODYSSEY}

The design of GRaND was based on experience from two successful planetary science missions. The GRaND BGO gamma ray sensor has approximately the same volume, detection efficiency, and pulse height resolution as the LPGRS. The neutrons sensors for the Dawn GRaND were selected to be similar in size and efficiency to the Mars Odyssey neutron spectrometer. Consequently, the data products from Dawn will be similar in many respects to those from Mars Odyssey and Lunar Prospector. The results of these missions are summarized here.

Lunar Prospector (1998-1999) flew boom-mounted gamma ray and neutron spectrometers. Data were acquired in a polar mapping orbit to obtain full coverage of the moon, including a low altitude $(30 \mathrm{~km})$ mapping orbit for 220 days and a high altitude $(100 \mathrm{~km})$ mapping orbit for 300 days. Maps of the global distribution of hydrogen were made using epithermal neutrons. This data set revealed water ice cold-trapped in permanently shadowed craters at the north and south pole [Feldman et al., 2001]. Fast neutron data were used to map the average atomic mass of the surface. Maps of the sum of rare earth elements Gd and Sm were also developed from neutron data combined with Clementine spectral reflectance data [Elphic et al., 2000].

The LPGRS data were reduced to determine global maps of major elements, including $\mathrm{O}, \mathrm{Mg}, \mathrm{Al}, \mathrm{Si}, \mathrm{Ca}, \mathrm{Ti}$, and $\mathrm{Fe}$, and radioactive elements, including $\mathrm{K}$ and Th. These were submitted to the Planetary Data System (PDS) archive for use by the lunar science community. Examples of map products are shown in Fig. 3. The maps were based on data acquired at high altitude, which was binned on $5^{\circ}$ equal area pixels. Higher resolution maps have also been developed using low altitude mapping data for $\mathrm{Fe}$ and $\mathrm{Th}$ and are available from the PDS. The Fe map was determined from capture and inelastic gamma rays in combination with neutron data, which was used to determine the equilibrium neutron number density in the surface. The Th map was determined from the $2.6 \mathrm{MeV}$ gamma ray produced by radioactive decay.

Both Th and Fe are concentrated in the near-side lunar maria. The distribution of these elements and the other rockforming elements help constrain models of lunar internal composition and thermal evolution. Fig. 4 shows correlation plots for LPGRS elemental data binned on $5^{\circ}$ pixels and lunar sample data for soils acquired by the Apollo and Luna missions. The close general comparison between the LPGRS and sample data help validate the analysis of the LPGRS data set. Outliers from the sample data, such as the high-Fe basalts in western Oceanus Procellarum, demonstrate the usefulness of gamma ray remote sensing data for geochemistry studies on a global scale. The scatter plot in Fig. 4b, for example, was used to classify non-mare regolith materials, resulting in the identification of highlands regions that differ significantly from the sample data [Prettyman et al., 2002].

A deck-mounted neutron spectrometer was flown on Mars Odyssey (2001-present). Odyssey entered into a polar mapping orbit at $400 \mathrm{~km}$ altitude. Over a martian year of mapping has been completed and an extended mission has begun. The spectrometer consists of boron-loaded plastic scintillator that was segmented into four prisms, each of which were viewed by separate photomultiplier tubes. The prism data were combined to determine thermal, epithermal, and fast neutron leakage flux from Mars. The relative motion of the space craft to neutrons crossing the orbital altitude of the spacecraft was exploited in determining the thermal neutron count rates. 

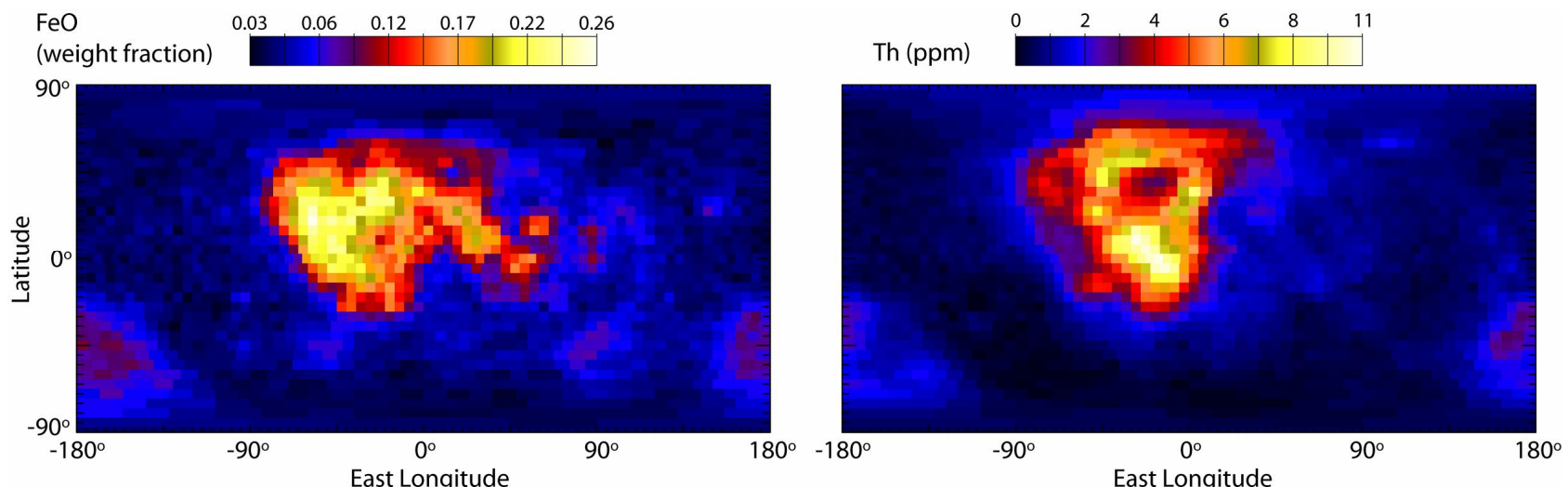

Figure 3. Global elemental maps of the moon determined from LPGRS data (from the Planetary Data System).
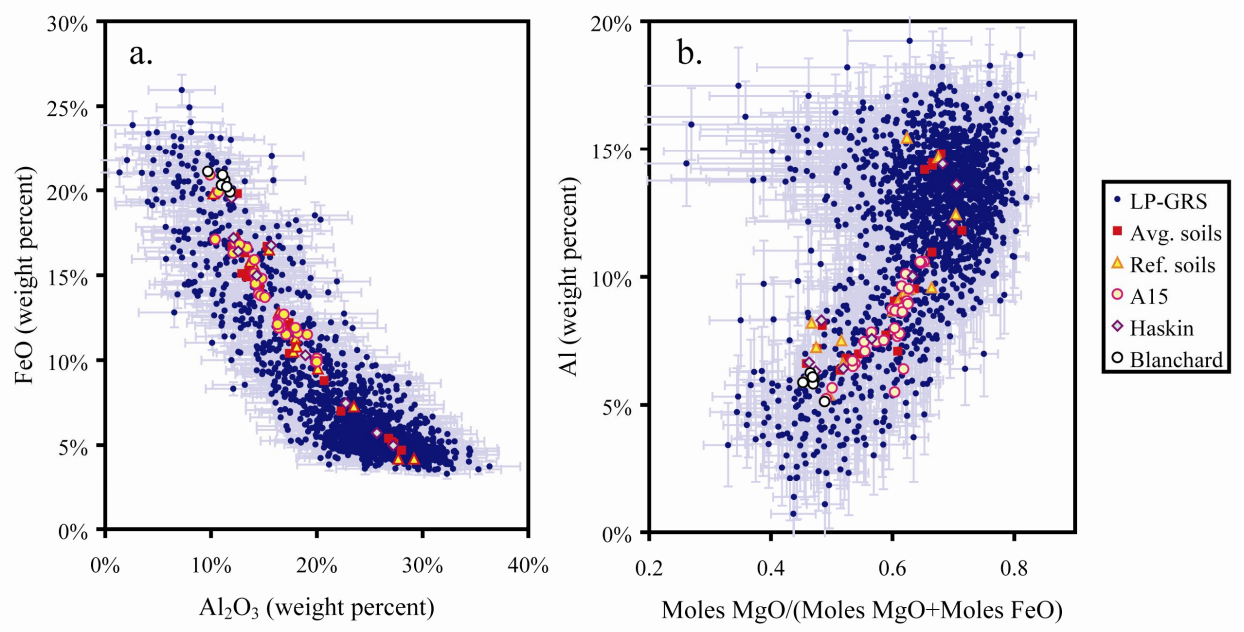

Figure 4. Comparison of LPGRS elemental data to lunar sample data acquired by the Apollo and Luna missions.

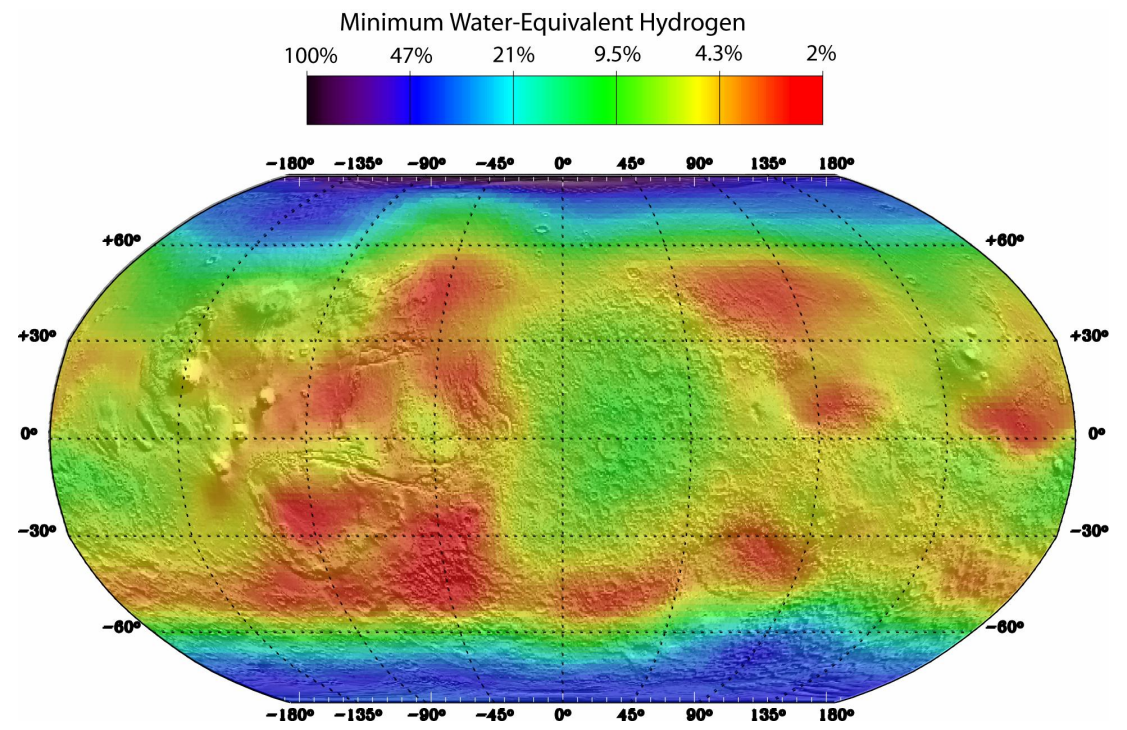

Figure 5. Map of water equivalent hydrogen on Mars. 
Data from the Odyssey neutron spectrometer was used to map the global abundance of water within about $1 \mathrm{~m}$ of the martian surface [Feldman et al., 2004b]. A map of water equivalent hydrogen (WEH) based on epithermal neutron counting data binned on $2^{\circ}$ equal area pixels is shown in Fig. 5. The abundance of WEH, given in weight percent, is a lower bound. The WEH map is superimposed on a map of shaded relief. The map reveals high concentrations of WEH at high latitudes, where water ice is stable close to the surface. At mid- to equatorial latitudes, the abundance of WEH ranges from $2 \%$ to about $10 \%$ over large regions where water is not stable. The WEH at equatorial latitudes may be in the form of hydrated minerals [Feldman et al., 2004c].

At high southern latitudes, the neutron data have been analyzed to determine the depth and water abundance of the ice table [Prettyman et al., 2004a; and references therein]. The ice table depths determined from the neutron analysis are consistent with ice stability models; however, the abundance of water ice determined from neutron spectroscopy is larger than can generally be accommodated by the pore space of typical soils. This suggests that emplacement mechanisms other than vapor exchange between the atmosphere and surface were involved in forming the ice table. The mass of $\mathrm{CO}_{2}$ in the southern residual cap was also determined by the analysis of neutron data along with the amount of near surface water ice in the cap region. The neutron data were found to be strongly sensitive to the presence of seasonal frost at high latitudes. Time dependent maps of the polar seasonal caps have been determined from the neutron data along with measurements of the enrichment and depletion of noncondensable gasses $\left(\mathrm{N}_{2}+\mathrm{Ar}\right)$, which are strong thermal neutron absorbers, in the polar atmosphere [Prettyman et al., 2004b; Prettyman and Titus, 2004c]. In summary, the data from the neutron spectrometer have provided powerful new constraints on martian climate history, the general circulation of the atmosphere, and local atmospheric dynamics.

\section{EXPECTED PERFORMANCE: SENSITIVITY TO ALTITUDE AND DURATION}

In order to achieve results comparable to Lunar Prospector and Mars Odyssey, the altitude and duration of the mapping orbits at Vesta and Ceres must be optimized. The gamma ray and neutron "signal" from the asteroid varies in proportion to the solid angle subtended by the asteroid at the detector, which decreases with altitude. At large distances from the body, the only contribution that can be observed is background radiation from the space environment. Low altitudes are clearly preferred; however, safety and navigation issues provide constraints on what can be achieved in practice. The duration of the mapping phase controls statistical precision and coverage. Altitude also governs the spatial resolution that can be achieved. The spatial resolution or footprint of the spectrometer varies roughly in proportion to altitude.

Bearing these issues in mind, what will we see while in mapping orbit around Vesta and Ceres? Vesta is similar to the moon in that it is expected to be a dry body. Although the mineralogy of Vesta is different from the moon, the same suite of major elements can be found on Vesta's surface. Consequently, this question can be answered for Vesta using data acquired by the LPGRS acquired immediately following orbital insertion.

Following capture, LP entered into a 12h elliptical orbit, which sampled a range of altitudes (Fig. 6). The solid angle of Vesta at the mapping altitude for the baseline mission $(200 \mathrm{~km})$ is the same as the solid angle of the moon at an altitude of $1300 \mathrm{~km}$. In order to simulate a spectrum from Vesta, we binned LPGRS data acquired near $1300 \mathrm{~km}$ altitude. The resulting pulse height spectrum is compared in Fig. 6 to the background pulse height spectrum acquired during cruise. The background is caused by the interaction of energetic particles and cosmic rays from the space environment and induced radioactivity in the LPGRS. The spectrum measured at $1300 \mathrm{~km}$ is well above background and shows spectral features associated with the lunar surface including gamma rays from $\mathrm{O}$ and $\mathrm{Fe}$. The integration time was $2.4 \mathrm{~h}$, which can be compared to a total integration time of 50 days at Vesta. From this analysis, we conclude that the altitude and duration for LAMO at Vesta are sufficient to meet our objectives for elemental abundance mapping.

Will GRaND be able to resolve surface features on Vesta and Ceres? The spatial response of GRaND while in orbit around Vesta is given as a function of altitude in Fig. 7. Each curve gives the contribution of gamma rays or neutrons from the surface as a function of distance from the sub-satellite point for a selected altitude. For $200 \mathrm{~km}$ altitude, the full-width-at-half-maximum (FWHM) of the response function is approximately $300 \mathrm{~km}$, which is on the scale of the great south polar crater (shown on the right of Fig. 7). However, a portion of the gamma rays and neutrons detected by 


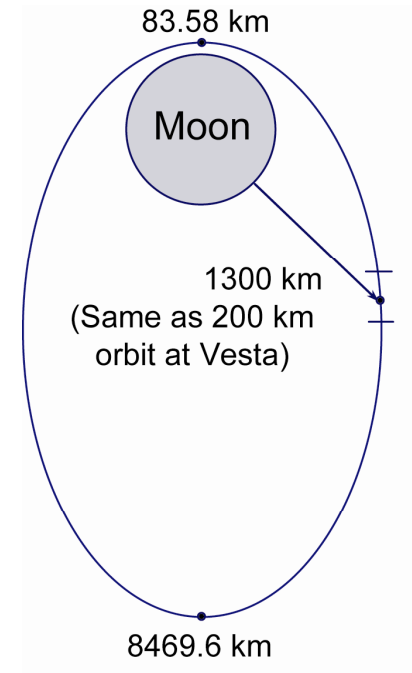

LP Capture Orbit (12h)

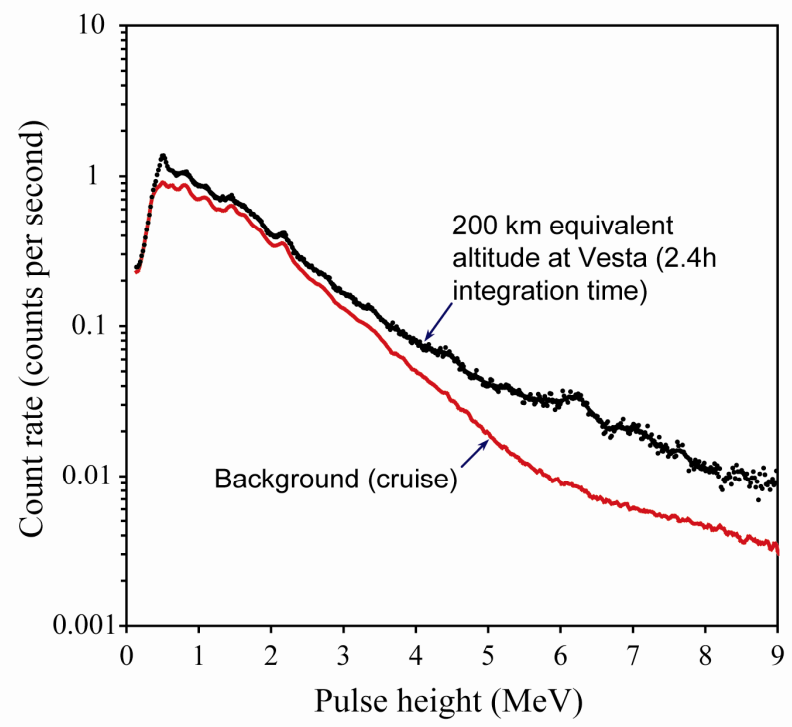

Figure 6. LPGRS lunar pulse height spectrum acquired at an altitude of $1300 \mathrm{~km}$, which is equivalent to $200 \mathrm{~km}$ altitude around Vesta.
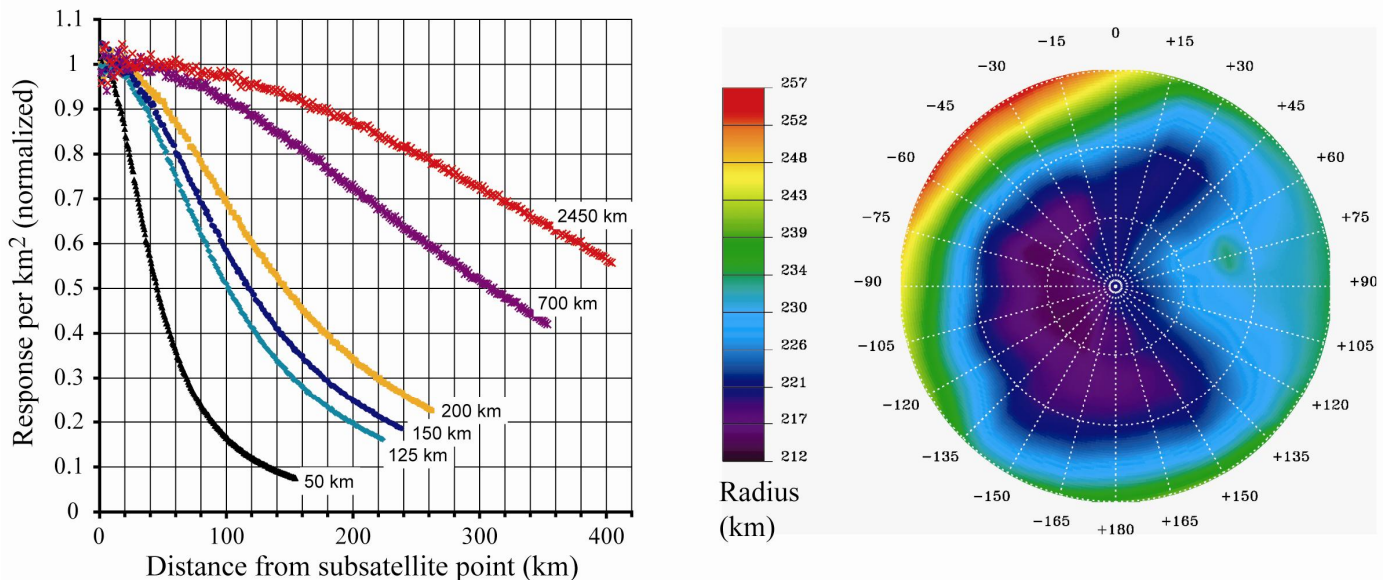

Figure 7. The spatial response of GRaND is shown for different altitudes at Vesta on the left. On the right, a stereographic projection $\left(45^{\circ} \mathrm{S}\right.$ to $\left.90^{\circ} \mathrm{S}\right)$ of the Vesta shape model shows the great south polar crater, which is roughly $200 \mathrm{~km}$ in diameter.

GRaND will come from terrain outside the crater. At $200 \mathrm{~km}$ altitude, accurate determination of the composition of the material inside the crater will require spatial deconvolution of the gamma ray and neutron data.

Analysis of spatial mixing to determine the composition of geographical units that are smaller in scale than the spectrometer footprint was demonstrated for 2001 Mars Odyssey [Prettyman et al., 2004a]. The spatial footprint of the Odyssey neutron spectrometer is $10^{\circ}$ arc length, FWHM. At this scale, the south polar residual cap cannot be resolved. In addition, the $\mathrm{CO}_{2}$ in the cap is a strong source of neutrons that interferes with observations of the surrounding water rich terrain. Using a combination of geographical and geophysical constraints, we separated the contribution of the cap from the surrounding terrain to simultaneously constrain cap composition and the properties of the adjacent water-rich material. 
The same approach will be applied to the analysis of counting data from Ceres and Vesta. High resolution imaging data from FC and VIR will be used to identify major geographical regions over which the composition can be assumed to be constant. Models of the instrument response that account for spatial mixing over the footprint will be used to estimate neutron and gamma ray count rate maps for trial regional compositions. Least squares or maximum likelihood analyses can be used to determine the composition of the regions. This approach will provide useful results as long as the regions selected are not significantly smaller than the footprint FWHM. Direct deconvolution methods that do not require the use of FC or VIR data will also be investigated; however, the use of a priori information is always the best approach when such information is available. Additional constraints on geochemistry could also be provided by mineralogical data acquired by VIR, including sums of major oxides, and elemental mixing models based on mineralogy.

\section{CONCLUSIONS}

GRaND will acquire global maps of the abundance of major rock forming elements, radioactive elements, and light elements such as H, C, and N at Vesta and Ceres. Dawn's baseline mission will enable elemental composition to be determined for at least 40 spatial regions on Vesta and at least 10 regions on Ceres. Data from FC and VIR will be used to define geographic regions for GRaND analysis. Application of spatial mixing and deconvolution algorithms will enable accurate analysis of regions that are smaller than the footprint of the instrument. The data acquired by GRaND will constrain the thermal history and geochemistry of both Vesta and Ceres, provide context for the HED meteorites at Vesta, and determine the role of water in planetary evolution.

\section{ACKNOWLEDGEMENTS}

This work was carried out by the Los Alamos National Laboratory (LANL), which is operated by the University of California for the U.S. Department of Energy under contract W-7405-ENG-36. The GRaND team consists of technical staff from the LANL's International Space and Response Division. Team members include Juan Baldonado, Bruce Barraclough, John Bernardin, David Cronk, Bob Dingler, Don Enemark, Danny Everett, Bill Feldman, Ken Fuller, Celestino Gonzales, Irma Gonzales, Jerry Kolar, Cindy Little, Ruxanne Lopez, Susan Nava, Doug Patrick, Tom Prettyman, James Sheldon, Gary Smith, Steve Storms, Vernon Vigil, and Bob Williford. The BGO sensor was provided by Proteus, Inc (Phil Parkhurst). The plastic and phoswich scintillators were manufactured by Eljen Technology (Chuck Hurlbut). The CdZnTe sensors were manufactured by eV Products (Steve Soldner and Csaba Czeles). Funding for the GRaND instrument was provided by NASA via contract no. DE-FI04-04AL67995. Chris Russell, professor of Geophysics and Space Physics at UCLA, is the principal investigator of the Dawn mission. More information about the Dawn mission can be found at http://dawn.jpl.nasa.gov/.

\section{REFERENCES}

Coradini, A. et al., 1998. VIRTIS: an imaging spectrometer for the Rosetta mission, Planet. Space. Sci., 46, 9/10, 12911304.

Elphic, R. C., D. J. Lawrence, W. C. Feldman, B. L. Barraclough, S. Maurice, A. B. Binder, P. G. Lucey, Lunar rare earth element distribution and ramifications for $\mathrm{FeO}$ and $\mathrm{TiO} 2$ : Lunar Prospector neutron spectrometer observations, Journal of Geophysical Research, Vol. 105, No. E8, pp. 20,333-20,345, August 25, 2000.

Feldman, W. C., D. J. Lawrence, R. C. Elphic, D. T. Vaniman, D. R. Thomsen, B. L. Barraclough, S. Maurice, A. B. Binder, Chemical information content of lunar thermal and epithermal neutrons, Journal of Geophysical Research, Vol. 105, No. E8, pp. 20,347-20,363, August 25, 2000.

Feldman, W. C., S. Maurice, D. J. Lawrence, R. C. Little, S. L. Lawson, O. Gasnault, R. C. Wiens, B. L. Barraclough, R. C. Elphic, T. H. Prettyman, J. T. Steinberg, and A. B. Binder, Evidence for water ice near the lunar poles, Journal of Geophysical Research, Vol. 106, No. E10, pp. 23,231-23,251, Oct. 25, 2001.

Feldman, W. C., K. Ahola, B. L. Barraclough, R. D. Belian, R. K. Black, R. C. Elphic, D. T. Everett, K. R. Fuller, J. Kroesche, D. J. Lawrence, S. L. Lawson, J. L. Longmire, S. Maurice, M. C. Miller, T. H. Prettyman, S. A. Storms, 
G. W. Thornton, Gamma-Ray, Neutron, and Alpha-Particle Spectrometers for the Lunar Prospector mission, Journal of Geophysical Research, VOL. 109, E07S06, doi:10.1029/2003JE002207, 2004a.

Feldman, W. C., T. H. Prettyman, S. Maurice, J. J. Plaut, D. L. Bish, D. T. Vaniman, M. T. Mellon, A. E. Metzger, S. W. Squyres, S. Karunatillake, W. V. Boynton, R. C. Elphic, H. O. Funsten, D. J. Lawrence, and R. L. Tokar, Global distribution of near-surface hydrogen on Mars, Journal of Geophysical Research, Vol. 109, E09006, doi:10.1029/2003JE002160, 2004b.

Feldman, W. C., M. T. Mellon, S. Maurice, T. H. Prettyman, J. W. Carey, D. T. Vaniman, D. L. Bish, C. I. Fialips, S. J. Chipera, J. S. Kargel, R. C. Elphic, H. O. Funsten, D. J. Lawrence, and R. L. Tokar, Hydrated states of $\mathrm{MgSO}_{4}$ at equatorial latitudes on Mars, Geophysical Research Letters, Vol. 31, L16702, doi:10.1029/2004GL020181, 2004c.

Gasnault, O., W. C. Feldman, S. Maurice, I. Genetay, C. d'Uston, T. H. Prettyman, and K. R. Moore, Composition from fast neutrons: Application to the Moon, Geophys. Res. Lett., 28(19), 3797- 3800, 2001.

Lawrence, D. J., W. C. Feldman, R. C. Elphic, R. C. Little, T. H. Prettyman, S. Maurice, P. G. Lucey, A. B. Binder, Iron abundances on the lunar surface as measured by the Lunar Prospector gamma-ray and neutron spectrometers, Journal of Geophysical Research, Vol. 107, No. E12, 5130, doi:10.1029/2001JE001530, 2002.

Lawrence, D. J., S. Maurice, W. C. Feldman, Gamma-ray measurements from Lunar Prospector: Time series data reduction for the gamma-ray spectrometer, Journal of Geophysical Research (JUN 9 2004) v.109, no.E7, p.E07S05.

Maurice S., W. C. Feldman, D. J. Lawrence, R. C. Elphic, O. Gasnault, C. d'Uston, I. Genetay, P. G. Lucey, Highenergy neutrons from the Moon, Journal of Geophysical Research, Vol. 105, No. E8 (2000) pp. 20365-20375.

Mustard, J.F., et al., 1989. Photometric phase functions of common geologic minerals and applications to quantitative analysis of mineral mixture reflectance spectra. J. Geophys. Res. 94, 13,619 - 13,634 NOB1 Opag.

Prettyman, T. H., D. J. Lawrence, D. T. Vaniman, R. C. Elphic, and W. C. Feldman, Classification of regolith materials from Lunar Prospector data reveals a magnesium-rich highland province," Workshop on the Moon Beyond 2002: Next Steps in Lunar Science and Exploration, September 12-14, 2002, Taos, New Mexico.

Prettyman, T. H., Feldman, W. C., Fuller, K. R., Storms, S. A., Soldner, S. A, Szeles, Cs., Ameduri, F. P., Lawrence, D. J., Browne, M. C., Moss, C. E., CdZnTe Gamma-Ray Spectrometer for Orbital Planetary Missions, IEEE Transactions on Nuclear Science, Vol. 49, 1881-1886, 2002.

Prettyman, T. H., W. C. Feldman, F. P. Ameduri, B. L. Barraclough, E. W. Cascio, K. R. Fuller, H. O. Funsten, D. J. Lawrence, G. W. McKinney, C. T. Russell, S. A. Soldner, S. A. Storms, Cs. Szeles, R. L. Tokar, Gamma-Ray and Neutron Spectrometer for the Dawn Mission to 1 Ceres and 4 Vesta, IEEE Transactions on Nuclear Science, Vol. 50, No. 4 (August 2003) pp. 1190-1197.

Prettyman, et al., W. C. Feldman, M. T. Mellon, G. W. McKinney, W. V. Boynton, S. Karunatillake, D. J. Lawrence, S. Maurice, A. E. Metzger, J. R. Murphy, S. W. Squyres, R. D. Starr, and R. L. Tokar, Composition and structure of the Martian surface at high southern latitudes from neutron spectroscopy, Journal of Geophysical Research, Vol. 109, E05001, doi:10.1029/2003JE002139, 2004a.

Prettyman, T. H., R. C. Wiens, J. R. Murphy, J. M. Reisner, and W. C. Feldman, Seasonal cycle of carbon dioxide and atmospheric circulation in Mars' southern hemisphere as observed by neutron spectroscopy, Lunar and Planetary Science XXXV (2004), Abstract 1878.

Prettyman, T. H. and T. N. Titus, Exploring Martian Polar Atmospheric Circulation and Surface Interactions, Eos, Vol. 85, No. 41, 12 October 2004c.

Reedy, R. C., J. R. Arnold, J. I., Trombka, Expected gamma ray emission spectra from the lunar surface as a function of chemical composition, Journal of Geophysical Research (10 Sept. 1973) Vol.78, No. 26, p.5847-66.

Russell, C. T., A. Coradini, U. Christensen, M.C. De Sanctis, W.C. Feldman, R. Jaumann, H.U. Keller, A.S. Konopliv, T.B. McCord, L.A. McFadden, H.Y. McSween, S. Mottola, G. Neukum, C.M. Pieters, T.H. Prettyman, C.A. Raymond, D.E. Smith, M.V. Sykes, B.G. Williams, J. Wise, M.T. Zuber, Dawn: A journey in space and time, Planetary and Space Science 52 (2004) $341-365$.

Treiman, A. H., A. Lanzirotti, D. Xirouchakis, Ancient water on asteroid 4 Vesta: evidence from a quartz veinlet in the Serra de Mage eucrite meteorite, Earth and Planetary Science Letters 219 (2004) 189-199. 